\title{
NATURALnESS AND THEORETICAL CONSTRAints ON THE HigGS BOSON MASS
}

\author{
A. R. Vieira • Brigitte Hiller - M. C. Nemes • Marcos Sampaio
}

Received: date / Accepted: date

Abstract Arbitrary regularization dependent parameters in Quantum Field Theory are usually fixed on symmetry or phenomenology grounds. We verify that the quadratically divergent behavior responsible for the lack of naturalness in the Standard Model (SM) is intrinsically arbitrary and regularization dependent. While quadratic divergences are welcome for instance in effective models of low energy QCD, they pose a problem in the SM treated as an effective theory in the Higgs sector. Being the very existence of quadratic divergences a matter of debate, a plausible scenario is to search for a symmetry requirement that could fix the arbitrary coefficient of the leading quadratic behavior to the Higgs boson mass to zero. We show that this is possible employing consistency of scale symmetry breaking by quantum corrections. Besides eliminating a finetuning problem and restoring validity of perturbation theory, this requirement allows to construct bounds for the Higgs boson mass in terms of $\delta m^{2} / m_{H}^{2}$ (where $m_{H}$ is the renormalized Higgs mass and $\delta m^{2}$ is the 1-loop

\section{A. R. Vieira}

Federal University of Minas Gerais, Physics Department, ICEx, PO Box 702, 30.161-970, Belo Horizonte MG, Brazil

E-mail: arvieira@fisica.ufmg.br

Brigitte Hiller

Coimbra University - Faculty of Science and Technology- Physics Department - Center of Computational Physics, Rua Larga, P3004-516 Coimbra - Portugal

E-mail: brigitte@teor.fis.uc.pt

M. C. Nemes

Federal University of Minas Gerais, Physics Department, ICEx, PO Box 702, 30.161-970, Belo Horizonte MG, Brazil

E-mail: carolina@fisica.ufmg.br

Marcos Sampaio

Federal University of Minas Gerais, Physics Department, ICEx, PO Box 702, 30.161-970, Belo Horizonte MG, Brazil

E-mail: msampaio@fisica.ufmg.br
Higgs mass correction). Whereas $\delta m^{2} / m_{H}^{2}<1$ (perturbative regime) in this scenario allows the Higgs boson mass around the current accepted value, the inclusion of the quadratic divergence demands $\delta m^{2} / m_{H}^{2}$ arbitrarily large to reach that experimental value.

PACS 14.80.Bn $\cdot$ 11.15.Bt $\cdot 11.10 . \mathrm{Gh}$

Keywords Naturalness · Fine-Tuning problem · Higgs mass · Implicit Regularization

\section{Introduction}

The Higgs field in the Standard Model (SM) is fundamental to ensure its renormalizability and unitarity. However, as pointed out by Susskind [1, there are selfenergy corrections to the Higgs propagator which would require an incredible fine-tuning of $10^{-34}$ parts in 1 , if we assume that the limit of validity of the SM is at the Planck scale. This problem does not appear in other theories which present Naturalness. The concept of Naturalness was defined by Susskind as when the behavior of the world at ordinary energies is not exceedingly sensitive to the values of the fundamental parameters [2]. Theories with scalar fields present unnaturalness because the mass of the scalar, a phenomenological parameter, exceedingly depends on the cutoff of the theory. In the context of the Standard Model, we can see this, after a regularization and a renormalization scheme, via the corrected mass of the Higgs boson [3, 8]

$$
\begin{aligned}
m_{H}^{2}= & m^{2}+\frac{3 \Lambda^{2}}{8 \pi^{2} v^{2}}\left[m_{Z}^{2}+2 m_{W}^{2}+m^{2}-4 m_{t}^{2}\right]+ \\
& +O\left(\ln \frac{\Lambda}{m}\right)
\end{aligned}
$$


where $m_{Z}, m_{W}, m$ and $m_{t}$ stand for $Z^{0}, W^{ \pm}$, Higgs and top quark masses, respectively, and $\Lambda$ is the energy scale of the theory. We see that large corrections, unnaturalness and the consequent fine-tuning problem are all caused by $\delta m^{2}=m_{H}^{2}-m^{2}$ being proportional to the energy scale squared.

Therefore, proposals to eliminate this quadratic energy scale have been put forth $[3,4,5,6$. One of them consisted in choosing the sum in square brackets in eq. (1) to be zero [3]. This gives a constraint among the masses. However, its validity at any energy scale is not guaranteed [4,9]. Such constraint overestimates the Higgs boson mass [8] as compared with the expected value 7 .

In ref. 4 is argued that the $\Lambda^{2}$ dependence of equation (1) is not in consonance with scale symmetry breaking. In order to see this consider the classical energymomentum tensor which is broken by the mass term

$\left.\Theta_{\mu}^{\mu}\right|_{\text {classical }}=m^{2} \bar{H} H$.

where $H$ denotes the Higgs field.

We have a symmetry in equation (2) in the limit $m \rightarrow 0$. The quantum corrections also break that conservation law:

$$
\begin{gathered}
\left.\Theta_{\mu}^{\mu}\right|_{1-\text { loop }}=\delta m^{2} \bar{H} H+\sum_{i} \frac{\partial \mathcal{L}}{\partial \lambda_{i}} \beta_{\lambda_{i}} \\
\delta m^{2}=\frac{3 \Lambda^{2}}{8 \pi^{2} v^{2}}\left[m_{Z}^{2}+2 m_{W}^{2}+m^{2}-4 m_{t}^{2}\right]+ \\
+O\left(\ln \frac{\Lambda}{m}\right)
\end{gathered}
$$

where $\beta_{\lambda_{i}}$ is the beta function associated with the coupling $\lambda_{i}$.

If we try to restore the classical limit, taking the limits $m \rightarrow 0$ and $\beta_{\lambda_{i}} \rightarrow 0$ in equation (3), the result is

$$
\left.\Theta_{\mu}^{\mu}\right|_{1-l o o p}=\left[\frac{3 \Lambda^{2}}{8 \pi^{2} v^{2}}\left(m_{Z}^{2}+2 m_{W}^{2}-4 m_{t}^{2}\right)\right] \bar{H} H
$$

Equation (4) shows that we do not restore the classical limit $\left(\Theta_{\mu}^{\mu}=0\right)$ when we take the classical limit $\left(m \rightarrow 0\right.$ and $\left.\beta_{\lambda_{i}} \rightarrow 0\right)$. In the limit where $m$ goes to zero, in order to preserve the structure of the anomalous divergence of the scale current, $\delta m^{2}$ must scale as $m^{2}$ rather than $\Lambda^{2}$. The conclusion is that this $\Lambda^{2}$ dependence is incompatible with the consistency of scale symmetry breaking, which leads us to analyze the possibility and implications of it being an artifact of regularization. In fact in eq. (3) the $\Lambda^{2}$ dependence results from the use of a sharp cutoff regularization, which breaks scale symmetry but not in a physical way, i.e. not related with the beta functions. The scheme causes spurious divergences.

A similar conclusion is drawn in ref. 5, using the Taylor-Lagrange renormalization. Furthermore in the Wilsonian renormalization group approach it was argued in ref. [6] that the consistency of scale invariance breaking of the SM is an alternative solution to the Naturalness problem.

In this work we show in a regularization independent way that the coefficient of the quadratic divergence which gives rise to the Naturalness problem is intrinsically arbitrary and should be set to zero on the grounds of the symmetry arguments discussed above. This is in consonance with the approach proposed in ref. [10, in which arbitrary parameters stemming from perturbation theory calculations should be fixed by the underlying symmetries of the model.

As we will explicitly show in the next section, a quadratic divergence can be generally parametrized as $I_{\text {quad }}\left(\mu^{2}\right)=\frac{i}{(4 \pi)^{2}}\left[c_{2} \Lambda^{2}+\left(1+c_{1}\right) \mu^{2}+\mu^{2} \ln \frac{\Lambda^{2}}{\mu^{2}}\right]$, where $c_{2}$ is an arbitrary regularization dependent constant.

Moreover, after these considerations, we can reestablish perturbation theory choosing the ratio $\delta m^{2} / m_{H}^{2}$ in a range between 0 to 1 . We obtain bounds for the Higgs boson mass in a $m_{H}$ versus $\Lambda$ diagram. We compare our bounds with those existent in the literature [11, 12, 13.

\section{Implicit Regularization and arbitrariness in divergent amplitudes}

An ultraviolet (UV) divergent amplitude will be regularized implicitly 14. In other words, we assume the existence of a regulator function for the integral and we separate the regularization dependent content from the finite one by using the following identity

$$
\begin{aligned}
\frac{1}{\left[(k-l)^{2}-\mu^{2}\right]} & =\sum_{j=0}^{n-1} \frac{(-1)^{j}\left(l^{2}-2 l . k\right)^{j}}{\left(k^{2}-\mu^{2}\right)^{j+1}}+ \\
& +\frac{(-1)^{n}\left(l^{2}-2 l . k\right)^{n}}{\left(k^{2}-\mu^{2}\right)^{n}\left[(k-l)^{2}-\mu^{2}\right]}
\end{aligned}
$$

where $k$ is the internal momentum, $\mu$ is the mass of the internal particle and $n$ is chosen such that the denominator of an UV divergent integral does not contain an external momentum $l$. We get basic divergent integrals which are defined as

$$
\begin{aligned}
& I_{\text {quad }}\left(\mu^{2}\right) \equiv \int^{\Lambda} \frac{d^{4} k}{(2 \pi)^{4}} \frac{1}{\left(k^{2}-\mu^{2}\right)} \\
& I_{\text {log }}\left(\mu^{2}\right) \equiv \int^{\Lambda} \frac{d^{4} k}{(2 \pi)^{4}} \frac{1}{\left(k^{2}-\mu^{2}\right)^{2}}
\end{aligned}
$$

where the index $\Lambda$ means that we assume the existence of a regulator just in order to perform mathematical manipulations with the integrand.

For example, one of the corrections to the Higgs propagator (see figure 1) is rewritten using eq. (5) for $n=1$ 


$$
\begin{aligned}
& \int^{\Lambda} \frac{d^{4} k}{(2 \pi)^{4}} \frac{1}{\left(k^{2}-m^{2}\right)\left[(k-p)^{2}-m^{2}\right]}=I_{l o g}\left(m^{2}\right)+ \\
& +\int^{\Lambda} \frac{d^{4} k}{(2 \pi)^{4}} \frac{p^{2}-2 p . k}{\left(k^{2}-m^{2}\right)^{2}\left[(k-p)^{2}-m^{2}\right]}
\end{aligned}
$$

The second term in (8) is finite in the UV limit by power counting of the internal momentum.

The most general parametrization of the basic divergent integral $I_{l o g}\left(\mu^{2}\right)$ can be constructed as follows. By noting that

$$
\frac{\partial I_{l o g}\left(\mu^{2}\right)}{\partial \mu^{2}}=\frac{-i}{(4 \pi)^{2} \mu^{2}}
$$

and

$$
\frac{\partial I_{q u a d}\left(\mu^{2}\right)}{\partial \mu^{2}}=I_{l o g}\left(\mu^{2}\right),
$$

should be obeyed by any regularization scheme, a general parametrization with explicit scale dependence that satisfies $(9)$ and 10 is given by

$I_{l o g}\left(\mu^{2}\right)=\frac{i}{(4 \pi)^{2}}\left[\ln \frac{\Lambda^{2}}{\mu^{2}}+c_{1}\right]$

$I_{\text {quad }}\left(\mu^{2}\right)=\frac{i}{(4 \pi)^{2}}\left[c_{2} \Lambda^{2}+\left(1+c_{1}\right) \mu^{2}+\mu^{2} \ln \frac{\Lambda^{2}}{\mu^{2}}\right]$,

where $c_{1}$ and $c_{2}$ are dimensionless and arbitrary constants. Such arbitrary and regularization dependent constants are inherent to perturbation theory and can be fixed on symmetry grounds [10]. For example, gauge and momentum routing invariance in QED demands that quadratic surface terms, which are related to arbitrary constants like $c_{1}$ and $c_{2}$, should vanish [15]. There are also some examples of extended QED where gauge invariance is used to fix Lorentz-violating terms [16].

A plethora of regularization schemes have been constructed to be used where gauge invariant Dimensional Regularization may fail, namely in the so called dimensional specific theories among which super-symmetric, chiral and topological quantum field theories figure in. A natural question would be which basic properties should a method that does not resort to analytical continuation in the space-time dimension should retain in order to be invariant. We start by illustrating with simple examples following [17]. Let $\Delta$ be the superficial degree of divergence of a 1-loop integral where the momentum $k$ runs. Consider the following $\Delta=2$ integrals,

$A=\int_{k} \frac{k^{2}}{\left(k^{2}-\mu^{2}\right)^{2}}$, and

$B=I_{q u a d}\left(\mu^{2}\right)+\mu^{2} I_{l o g}\left(\mu^{2}\right)$,

where $\int_{k} \equiv \int d^{4} k /(2 \pi)^{4}$ and we recover the standard notation of eqs. 6 and 7 .

We expect $A=B$ be guaranteed by any regularization procedure. However this is not the case. Propertime regularization [18, for instance, introduces a cutoff $\Lambda$ after Wick rotation via the following identity at the level of propagators

$$
\begin{aligned}
\frac{\Gamma(n)}{\left(k^{2}+\mu^{2}\right)^{n}} & =\int_{0}^{\infty} d \tau \tau^{n-1} e^{-\tau\left(k^{2}+\mu^{2}\right)} \rightarrow \\
& \rightarrow \int_{1 / \Lambda^{2}}^{\infty} d \tau \tau^{n-1} e^{-\tau\left(k^{2}+\mu^{2}\right)} .
\end{aligned}
$$

Thus it is trivial to obtain within the proper-time method that $A \neq B$ since

$A_{\Lambda}=\frac{-2 i}{(4 \pi)^{2}}\left(\Lambda^{2}-\mu^{2} \ln \Lambda^{2} / \mu^{2}\right)$,

whereas

$B_{\Lambda}=\frac{-i}{(4 \pi)^{2}}\left(\Lambda^{2}-2 \mu^{2} \ln \Lambda^{2} / \mu^{2}\right)$.

As we can see above, an inadequate regularization scheme could fix the constants $c_{1}$ and $c_{2}$ in a way that would lead us to inappropriate conclusions. In the context of this work, the value $c_{2}=-1$, obtained with a sharp cutoff regularization, would lead to the finetuning problem. In the context of scalar QED coupled to gravitation, a non-vanishing value of $c_{2}$ would make this theory asymptotically free [19].

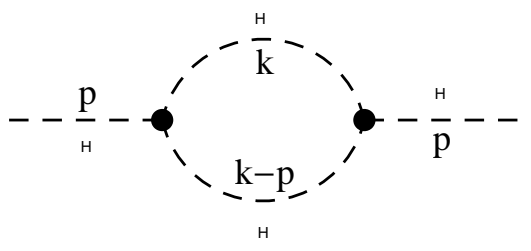

Fig. 1 One of the corrections to the Higgs propagator. 


\section{1-loop corrections to the Higgs propagator and theoretical bounds on the Higgs mass}

After spontaneous symmetry breaking, the contributions to the Higgs boson propagator include massive and Goldstone fields in the Landau gauge $(\xi=0)$.
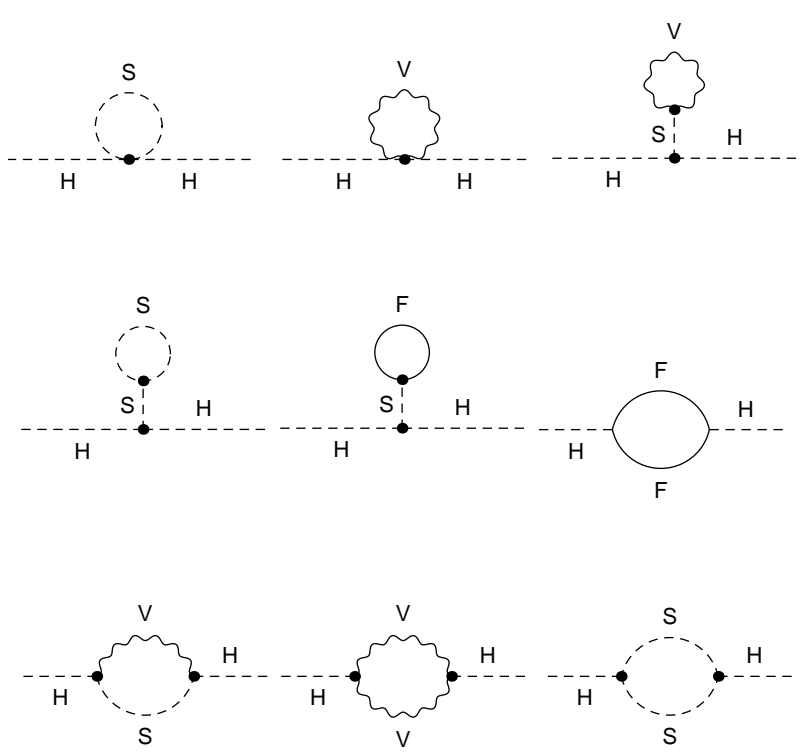

Fig. 2 1-Loop correction to the Higgs propagator in the Landau gauge. $\mathrm{S}, \mathrm{V}$ and $\mathrm{F}$ stands for scalar, vector boson and fermionic field, respectively

Figure 2 shows all 1-loop contributions. We also consider only the heaviest fermion ( top quark) for the fermionic 1-loop diagrams.

If one regularizes with a sharp covariant Euclidean cutoff and an on-mass-shell renormalization, for example, one has the Higgs mass correction given by equation (1). The quadratic cutoff of that equation means that we have an upper bound for this theory around $2 \mathrm{TeV}$ [9,21. This implies that the Standard Model, as an effective theory, should be valid up to energy scales that have already been reached by LHC [21]. Furthermore, one could expect the appearance of new physics around this energy, which so far did not emerge. So one can question whether the scale for the onset of new physics is really of that order. In addition, as the Higgs mass is expected to be $125 \mathrm{GeV}$ [7] we also would expect the cutoff to be much larger than that mass to preserve the hierarchy of the theory.

In terms of the basic divergent integrals defined in eqs. 11 and 12, the 1-loop contributions in figure 2 can be written in an on-mass-shell renormalization, for ex- ample, as

$$
\begin{aligned}
& \delta m^{2}=\frac{6 i}{v^{2}}\left[m_{Z}^{2}+2 m_{W}^{2}+m^{2}-4 m_{t}^{2}\right] I_{q u a d}\left(m^{2}\right)+ \\
& \frac{-3 i m^{2}}{v^{2}}\left[3 m^{2}+6 m_{W}^{2}+m_{Z}^{2}-6 m_{t}^{2}\right] I_{l o g}\left(m^{2}\right)
\end{aligned}
$$

where we neglect finite terms in the UV limit because they are small compared to terms proportional to $\Lambda$ and $\ln \Lambda$ when $\Lambda$ is large. For example, the finite integral in the UV limit of eq. (8) is much smaller than one when $p^{2}=m_{H}^{2}$.

Now, considering eq. [12, $\delta m^{2}$ correction reads

$\delta m^{2}=\frac{-3 c_{2}}{8 \pi^{2} v^{2}}\left[m_{Z}^{2}+2 m_{W}^{2}+m^{2}-4 m_{t}^{2}\right] \Lambda^{2}+\ldots$

where the ellipses stand for contributions from $I_{l o g}\left(m^{2}\right)$ and other terms from eq. 12 .

Because $c_{2}$ is arbitrary as discussed in section 2 it is consistent with a vanishing quadratic divergence. This assumption is justified by compatibility with scale symmetry breaking which at one loop can be broken only by terms proportional to the beta function and terms which vanish in the limit $m \rightarrow 0$ as argued in section 1. Thus it consists of a theoretical symmetry argument that fix an arbitrariness in this case.

The constant $c_{1}$ from eqs. (11) and 12 is also chosen using this argument, i. e. the finite part in the UV limit of the diagrams can not contain terms which are not proportional to $m^{2}$ because they break scale invariance in the limit $m \rightarrow 0$. These considerations complies with the definition of Naturalness imposed by 't Hooft [20].

A second argument from the phenomenological standpoint in favor of this choice is to assure Naturalness of the SM and the consequent absence of the fine-tuning problem. This choice appears to be more appealing than the one made in refs. [3] and [8], since now the quadratic energy scale vanishes without the need to enforce a constraint among masses of different particles which may vary differently with the energy scale.

There remain only the terms proportional to $m^{2} \ln \frac{\Lambda}{m}$ which do not break scale symmetry in the limit $m \rightarrow 0$. These terms come from the integrals $I_{\text {quad }}\left(\mathrm{m}^{2}\right)$ and $I_{l o g}\left(m^{2}\right)$ rewritten using eqs. (11) and $(12)$. So, the correction to the Higgs mass is given by

$\delta m^{2}=\frac{3 m_{H}^{2}}{16 \pi^{2} v^{2}}\left[2 m_{t}^{2}+2 m_{W}^{2}+m_{H}^{2}-m_{Z}^{2}\right] \ln \frac{\Lambda^{2}}{m_{H}^{2}}+\ldots$

where we have used $m_{H}^{2}=m^{2}+O\left(\frac{3 m^{2}}{16 \pi^{2} v^{2}}\right)$ to replace $m$ by $m_{H}$. The neglected terms are of order of two-loop corrections.

If we consider only contributions of the order $\ln \frac{\Lambda}{m_{H}}$, we can use experimental data for the masses $\left(m_{t}=\right.$ 
$173 \mathrm{GeV}, m_{W}=80.2 \mathrm{GeV}$ and $\left.m_{Z}=91.2 \mathrm{GeV}\right)$, the vev value $(v=246 \mathrm{GeV})$ and the currently accepted value for the Higgs mass $\left(m_{H}=125 \mathrm{GeV}\right)$, to estimate the limit of validity of perturbation theory. To do this, we ask where perturbation theory starts to fail, i. e. where $\delta m=O\left(m_{H}\right)$. We get $\Lambda \approx 10^{10} \mathrm{GeV}$.

We can obtain a more refined estimate if we consider running masses in equation 20). To include this information in that equation, we use the usual Standard Model relations between masses and coupling constants. Then we solve the beta functions of ref. [9] in order to find the running coupling constants. We have

$\frac{\delta m^{2}}{m_{H}^{2}}=\frac{3}{8 \pi^{2}}\left[\frac{m_{H}^{2}}{v^{2}}+\frac{1}{4} g^{2}(\Lambda)-\frac{1}{4} g^{\prime 2}(\Lambda)+g_{t}^{2}(\Lambda)\right] \ln \frac{\Lambda}{m_{H}}$

where $g(\Lambda), g^{\prime}(\Lambda)$ and $g_{t}(\Lambda)$ are the solutions of one loop beta functions for the $\mathrm{SU}(2), \mathrm{U}(1)$ and Yukawa couplings, respectively. We use as initial values $g^{2}\left(m_{Z}\right)=$ $0.42, g^{\prime 2}\left(m_{Z}\right)=0.13$ and $g_{t}\left(m_{t}\right)=1.01$.

The estimate now (for $v=246 \mathrm{GeV}$ and $m_{H}=$ $125 \mathrm{GeV})$ is $\Lambda \approx 10^{16} \mathrm{GeV}$. At this scale $\delta m$ starts to be equal or higher than $m_{H}$. That limit would be of order $1 \mathrm{TeV}$ should quadratic divergences be included [9,21].

Instead of assuming that perturbation theory remains valid up to the scale of $10^{16} \mathrm{GeV}$, one can alternatively present estimates for $\delta m^{2} / m_{H}^{2}$ in the range 0 to 1 where perturbation theory is still acceptable. A more complete and adequate analysis is to use eq. 21) plus the finite part in the UV limit to exclude a set of values of $m_{H}$ and $\Lambda$ that yields values of corrections outside the $\delta m^{2} / m_{H}^{2}$ value we have chosen. The result is a region of excluded values of the Higgs mass in a certain energy scale. Figure 3(a) shows a blue, a brown and a light green bound which excludes all values of $m_{H}$ and $\Lambda$ whose correction $\delta m^{2}$ is larger than or equal to $35 \%, 40 \%$ and $45 \%$ of the Higgs mass $m_{H}$, respectively. There is a pink bound which corresponds to the vacuum stability bound [11] that excludes all values of $m_{H}$ and $\Lambda$ that lead to a negative Higgs self-coupling. Our bounds also coincide in part with the triviality bound [12](dark green bound) which excludes all values of $m_{H}$ and $\Lambda$ which trespass the Landau pole. For $\delta m^{2} / m_{H}^{2} \sim 0.1$ we obtain bounds which essentially overlap with the vacuum stability bound and prevent the existence of allowed values for the Higgs mass. On the other hand, a bound $\delta m / m_{H} \rightarrow 1$ approaches the triviality bound and large ranges for $m_{H}$ are obtained.

For a given value of the mass correction, we can determine a Higgs mass range or a Higgs mass value in a certain energy scale. The crossing of the vacuum stability bound with a $\delta m^{2} / m_{H}^{2}$ percentage curve yields the minimal mass correction that one can achieve at

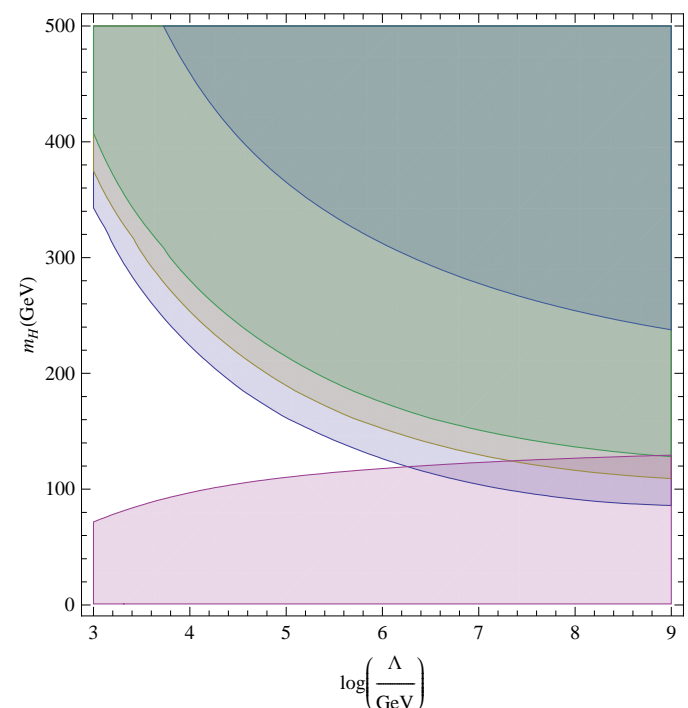

(a)

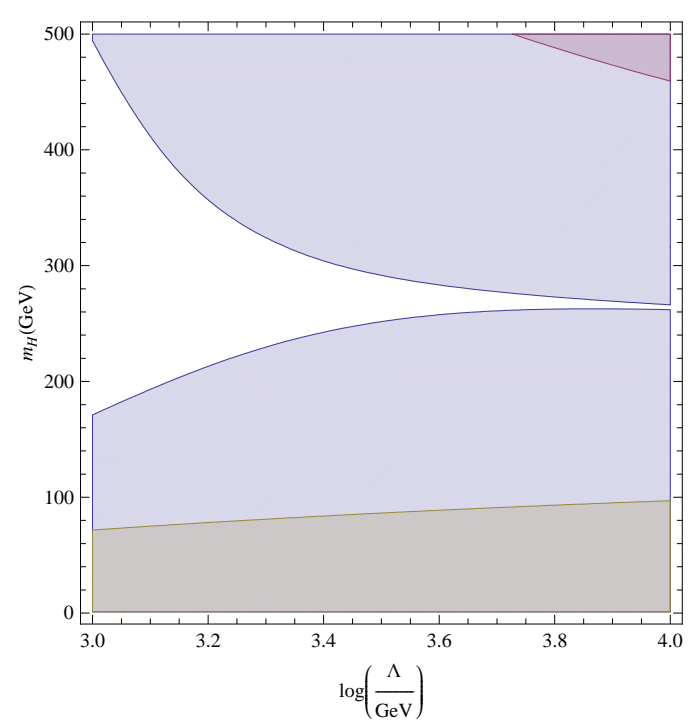

(b)

Fig. 3 (a)Excluded values for the Higgs mass: the pink bound is the vacuum stability bound [11] and the dark green one is the triviality bound [12]. The blue, brown and light green bounds are the ones which exclude all values of $m_{H}$ and $\Lambda$ which lead to corrections $\delta m^{2}$ larger than or equal to $35 \%, 40 \%$ and $45 \%$ of $m_{H}^{2}$, respectively.(b)Excluded values for the Higgs boson mass considering $\Lambda^{2}$ dependence: the brown bound is the vacuum stability bound [1] and the pink one is the triviality bound [12]. The blue bound is the one which excludes all values of $m_{H}$ and $\Lambda$ which lead to corrections $\delta m^{2}$ larger than or equal to $m_{H}^{2}$.

a certain scale. Its crossing with the blue mass region means that for a $35 \%$ correction and in a energy around $10^{3} \mathrm{TeV}$, the Higgs mass value is around $119 \mathrm{GeV}$. For the brown region, we have a Higgs mass value around $124 \mathrm{GeV}$ in an energy around $10^{4} \mathrm{TeV}$, if the mass correction squared is around $40 \%$. That means that if the cutoff of the SM is around that order we still have a 
Higgs boson mass near the expected one [7]. The same interpretation can be given for the light green bound. In that case, we obtain a Higgs mass around $129 \mathrm{GeV}$ for energies around $10^{6} \mathrm{TeV}$. We see that the greater the correction value, the greater the allowed regions for the Higgs mass. For example, the crossing of the bound obtained for a $20 \%$ correction with the vacuum stability bound occurs at $10 \mathrm{TeV}$ and we have a smaller white region than the ones of figure $3(\mathrm{a})$

The use of a consistent scale symmetry breaking to fix an arbitrary parameter that controls the quadratic divergence excludes the fine-tuning problem and consequently, we have a reliable perturbation theory. The choices $\delta m^{2}<m_{H}^{2}$ are also in agreement with the Renormalization Group because those bounds do not trespass the triviality bound. In the scenario where finetuning is used, the Higgs mass should approach $160 \mathrm{GeV}$ at an energy of $100 \mathrm{TeV}$ [13. However, the Higgs mass correction of ref. [13] violates perturbation theory and the consistency of the scale symmetry breaking.

Furthermore, these bounds give another argument against the $\Lambda^{2}$ dependence. If that dependence exists, the measured value of $125 \mathrm{GeV}$ is excluded for all energy scales and for all $\frac{\delta m^{2}}{m_{H}^{2}}<1$. We draw the bound corresponding to $\frac{\delta m^{2}}{m_{H}^{2}}=1$ (the maximum perturbation theory allows) considering the $\Lambda^{2}$ dependence in figure 3(b). That means if we want the Higgs mass around the expected value we must allow some finetuning, i.e. $\delta m^{2} / m_{H}^{2}>1$, as we can see in ref. [13]. On the other hand, the result of figure $3(\mathrm{a})$ allows Higgs masses around the expected value for $\delta m^{2} / m_{H}^{2}<1$, where perturbation theory is acceptable.

\section{Concluding remarks}

We showed that regularization dependent arbitrariness which appear in quadratic divergences can be parametrized as shown in section 2 and fixed on symmetry grounds to restore Naturalness and perturbation theory for the Higgs boson mass in the SM. We have analyzed the quantum corrections to the Higgs boson mass together with the vacuum stability bound. Although the correction values are not known, it is possible to establish bounds if we choose certain values for $\delta m^{2} / m_{H}^{2}$. These bounds provide phenomenological arguments against the existence of $\Lambda^{2}$ dependence in the Higgs boson mass correction.

\section{Acknowledgments}

Research supported by Fundação para a Ciêcia e Tecnologia, project CERN/FP/116334/2010, developed under the iniciative QREN, financed by UE/FEDER through COMPETE - Programa Operacional Factores de Competitividade. This research is part of the EU Research Infrastructure Integrating Activity Study of Strongly Interacting Matter (HadronPhysics3) under the 7th Framework Programme of EU: Grant Agreement No. 283286.

The authors thank CNPq and FAPEMIG for financial support and Dr. Luis Cabral for fruitful discussions.

\section{References}

1. L. Susskind Phys. Rev. D 202619 (1979)

2. L. Susskind Phys. Rep. 104181 (1984)

3. M. Veltman Acta Physica Polonica B 12437 (1981)

4. W. Bardeen 1995 FERMILAB-CONF-95-391-T

5. P. Grange, J.-F. Mathiot, B. Mutet and E. Werner PoS LC2010 027 (2012)

6. Hajime Aoki and Satoshi Iso Phys. Rev. D 86013001 (2012)

7. S. Chatrchyan et al. Phys. Lett. B 716 30-61 (2012)

8. G. L. Castro and J. Pestieau Mod. Phys. Lett. A 101155 (1995)

9. M. Chaichian, R. G. Felipe and K. Huitu Phys. Lett. B 363 101 (1995)

10. R. Jackiw Int. J. Mod. Phys. B 142011 (2000)

11. G. Altareli and G. Isidori Phys. Lett. B337 141 (1994); J. A. Casas, J. R. Espinosa, and M. Quiros Phys. Lett. B342 171 (1996); J. A. Casas, J. R. Espinosa, and M. Quiros Phys. Lett. B 382374 (1996).

12. N. Cabibbo, L. Maiani, G. Parisi and R. Petronzio Nucl. Phys. B 158295 (1979); R. Dashen and H. Neuberger Phys. Rev. Lett.50 1897 (1983); D. J. E. Callaway Nucl. Phys. B233 189 (1984); M. A. Beg, C. Panagiotakopolus, and A. Sirlin Phys. Rev. Lett. 52883 (1984); M. Lindner Z. Phys. C 31295 (1986); T. Hambye and K. Riesselmann Phys. Rev. D 557255 (1997)

13. C. Kolda and H. Murayama JHEP 07035 (2000)

14. O. A. Battistel, A. L. Mota and M. C. Nemes Mod. Phys. Lett. A 131597 (1998); Marcos Sampaio, A. P. Baeta Scarpelli, J. E. Ottoni, M. C. Nemes Int. J. of Theor. Phys. 45 436-457 (2006); C.R. Pontes, A.P. Baeta Scarpelli, Marcos Sampaio, J.L. Acebal, M.C. Nemes Eur. Phys. J C 53 121131 (2008); H. G. Fargnoli, B. Hiller, A. P. Baeta Scarpelli, Marcos Sampaio, M. C. Nemes Eur. Phys. J C71:1633 (2011)

15. L. C. Ferreira, A. L. Cherchiglia, Brigitte Hiller, Marcos Sampaio and M. C. Nemes Physical Review D 86, 025016(2012).

16. G. Gazzola, H. G. Fargnoli, A. P. Baeta Scarpelli, Marcos Sampaio and M. C. Nemes J. of Phys. G: Nucl. Part. Phys. 39035002 (2012); A. P. Baeta Scarpelli J. of Phys. G: Nucl. Part. Phys. 39125001 (2012)

17. T. Varin, D. Davesne, M. Oertel and M. Urban, Nucl. Phys. A 791422 (2007).

18. J. Zinn-Justin, Int. Ser. Monogr. Phys. 851 (1993).

19. D. J. Toms, Nature 468, 56 (2010); J. C. C. Fellipe, L. C. T. Brito, Marcos Sampaio, M. C. Nemes Phys. Lett. B 700 86 (2011); J.C.C. Felipe, L.A. Cabral, L.C.T. Brito, Marcos Sampaio and M.C. Nemes hep-th/1205.6779(2012)

20. G. 't Hooft Recent developments in Gauge Theories vol 59(NATO ASI Series B: Physics, Plenum Press, New York)(1979)

21. J. R. Espinosa Acta Physica Polonica B 38567 (2006) 